\title{
Predicting the Quality of Warfarin Therapy: Reframing the Question
}

\author{
Geoffrey D. Barnes, MD, MSc ${ }^{1}$ \\ ${ }^{1}$ Department of Internal Medicine, Frankel Cardiovascular Center, \\ University of Michigan, Ann Arbor, Michigan, United States \\ Thromb Haemost 2019;119:509-511.
}

Medical treatment of venous thromboembolism (VTE) is undergoing a paradigm shift. ${ }^{1}$ Before 2009 , vitamin $\mathrm{K}$ antagonists (VKAs) such as warfarin were the only widely available oral anticoagulants (OACs) for the acute and longterm treatment of VTE. In the decade since, four direct OACS (DOACs) have become the first-line therapy for many patients with VTE given their efficacy, favourable safety profile and ease of use. ${ }^{2}$ The same can be said for stroke prevention in patients with atrial fibrillation (AF), where DOACs have also become the first-line therapy. ${ }^{3}$

However, with more therapeutic choices comes the challenge of selecting the best therapy for each individual patient. For some patients, the choice is easy (e.g. VKA for patients with mechanical heart valves). However, for many patients, a variety of clinical and non-clinical factors may influence the decision process. It stands to reason that predicting the quality of VKA therapy may be useful when selecting between VKA and DOAC therapy. This is particularly important given the VKAs remain very widely used globally.

Initially developed in 2013 for warfarin-treated patients with AF, the SAMe-TT2R2 score incorporates many clinical and demographics factors into a risk score for good or poor warfarin control. ${ }^{4}$ Using data from the Atrial Fibrillation Follow-up Investigation of Rhythm Management trial, the authors who initially developed the SAMe-TT2R2 score described good discriminatory ability (c-index of $0.70-0.72$ ) for identifying which patients would experience extremely poor VKA control (time in the therapeutic range [TTR] of 30$35 \%$ ). However, the discriminatory ability was less robust (cindex of 0.58 ) for a more clinically relevant TTR threshold of 50 to $55 \%$. Similar results were seen in other validation studies of VKA-treated patients with AF ( - Table 1). $)^{5,6}$ Nonetheless, use of the SAME-TT2R2 score has been recommended as a possible guide for OAC decision-making for patients with AF. Specifically, patients with a score of $>2$ are less likely to achieve a good TTR. Therefore, they are recommended to receive early review, more frequent international normalized ratio checks and education or counselling to ensure safe and effective

received

February 7, 2019

accepted after revision

February 7, 2019

\author{
Address for correspondence Geoffrey D. Barnes, MD, MSc, \\ Department of Internal Medicine, University of Michigan, Frankel \\ Cardiovascular Center, Ann Arbor, MI, United States \\ (e-mail: gbarnes@umich.edu).
}

warfarin therapy. ${ }^{7}$ Otherwise, these patients can be considered for DOAC therapy instead of warfarin.

The study by Barco et al in this issue of Thrombosis and Haemostasis explores the predictive ability of the SAMe-TT2R2 score for VKA control in patients with VTE. ${ }^{8}$ This is a reasonable question given that many of the SAMe-TT2R2 elements apply to patients with AF and VTE (e.g. age, gender, race, tobacco use, co-morbidities). In their analysis of 3,874 patients with VTE treated with warfarin in the control arm of a randomized clinical trial, those with a low SAMe-TT2R2 score (0-1) represented a minority of patients in the cohort (24\%) and had a lower TTR than patients with a SAMe-TT2R 2 score of $\geq 2$ (64.7\% vs. $70.7 \%, p<0.001)$. However, they found that low negative $(0.59)$ and positive predictive ability ( 0.52$)$ and discriminatory characteristics (c-index 0.58 ) for a TTR cut-off of $66 \%$. These findings broadly mirror those from prior validation studies in VTE populations (-Table 1). ${ }^{9,10}$ Of note, many risk scores based on clinical factors have $c$-indexes near or under 0.6.

This is contrast to the practice-based observational study by Kataruka et al of 1,943 patients with newly diagnosed VTE being initiated on warfarin. ${ }^{8}$ In that study, patients with higher SAMe-TT2R2 scores had lower mean TTR $(57 \pm 21 \%$ vs. $50 \pm 23 \%$ for SAMe-TT2R2 or $0-1$ vs. $>3$ ). The discriminatory ability to predict a TTR of $65 \%$ was 0.65 , moderately higher than in the Barco et al study. This may reflect the overall lower quality of warfarin control in the practicebased cohort from Kataruka et al than the randomized trial cohort reported by Barco et al.

Certain factors may have influenced the utility of the SAMe-TT2R2 score in this analysis by Barco et al. First, they used a post hoc analysis of the Hokusai-VTE study, where patients with acute VTE were randomized to warfarin or edoxaban therapy following a 5- to 10-day parenteral lead in period. Using a highly selected randomized controlled trial population with specific inclusion and exclusion criteria may introduce selection bias in favour of patients who are healthier, more compliant with medical therapy and often have closer health system monitoring than unselected practice-

(c) 2019 Georg Thieme Verlag KG Stuttgart · New York
DOI https://doi.org/ 10.1055/s-0039-1681060. ISSN 0340-6245. 
Table 1 Performance of the SAME-TTR score in AF and VTE studies

\begin{tabular}{|c|c|c|c|c|}
\hline Study population & Study size & Study type & Outcome measure & c-Index $(95 \% \mathrm{Cl})$ \\
\hline \multirow[t]{2}{*}{$\begin{array}{l}\text { Atrial fibrillation } \\
\text { (derivation study) }\end{array}$} & \multirow[t]{2}{*}{1,019} & \multirow[t]{2}{*}{ Randomized trial } & TTR $<31 \%$ & $\begin{array}{l}0.72 \\
(0.64-0.79)\end{array}$ \\
\hline & & & TTR $<50 \%$ & $\begin{array}{l}0.58 \\
(0.53-0.62)\end{array}$ \\
\hline \multirow[t]{2}{*}{ Atrial fibrillation $^{5}$} & \multirow[t]{2}{*}{911} & \multirow[t]{2}{*}{ Practice-based, observational } & PINRR $\leq 70 \%$ & $\begin{array}{l}0.56 \\
(0.52-0.60)\end{array}$ \\
\hline & & & PINRR $\leq 60 \%$ & $\begin{array}{l}0.53 \\
(0.49-0.57)\end{array}$ \\
\hline Atrial fibrillation $^{6}$ & 1,524 & Practice-based, observational & TTR $<65 \%$ & $\begin{array}{l}0.56 \\
(0.53-0.59) \\
\end{array}$ \\
\hline Venous thromboembolism ${ }^{8}$ & 3,874 & Randomized trial & TTR $<66 \%$ & $\begin{array}{l}0.58 \\
(0.56-0.60)\end{array}$ \\
\hline Venous thromboembolism ${ }^{9}$ & 1,943 & Practice-based, observational & TTR $<65 \%$ & $0.65^{\mathrm{a}}$ \\
\hline Venous thromboembolism $^{10}$ & 1,308 & Practice-based, observational & TTR $<65 \%$ & $\begin{array}{l}0.52 \\
(0.48-0.55)\end{array}$ \\
\hline
\end{tabular}

Abbreviations: AF, atrial fibrillation; $\mathrm{Cl}$, confidence interval; PINRR, per cent of international normalized ratio results; TTR, time in the therapeutic range; VTE, venous thromboembolism.

${ }^{\mathrm{a}} 95 \%$ confidence interval not provided.

based patients. Second, anytime a risk score is used in a different population than it was initially derived (e.g. use in VTE while developed in AF), it may need adaptation to improve its discriminatory and predictive properties. Perhaps other elements are needed to predict VKA management quality that may be more specific to the VTE population.

Nevertheless, it is not clear that testing a summative risk score to predict good versus poor quality VKA therapy is the most useful clinical question. For many patients, DOAC therapy is the first line when clinically appropriate. However, for others, use of VKA is preferable due to drug cost, availability or concerns about readily available reversal agents. For these patients, identifying modifiable characteristics that may improve the quality of VKA therapy is a worthwhile clinical and research endeavour. Some of these characteristics are likely to be included in the SAMe-TT2R2 score, such as tobacco use and concurrent use of medications with drugdrug interactions. However, other elements of the SAMeTT2R2 score (e.g. age, race, sex) are not modifiable. And a direct connection between individual SAMe-TT2R2 score elements and VKA control is not always apparent. For instance, it follows logically that removing an interacting drug may improve the quality of VKA therapy. However, it is not clear how much tobacco use itself influences VKA control or if tobacco use is a marker for some other patient characteristic that influences VKA control.

Future studies aiming to improve anticoagulation care for patients with VTE are needed, including:

- How can risk scores be incorporated into decision aids that promote shared decision making for patients with AF and/or VTE?

- Which modifiable clinical factors associate with good/ poor VKA therapy? Which of these factors are specific to patients with AF and VTE?
- What interventions can be targeted at these modifiable clinical factors and how much improvement in the quality of VKA therapy can be expected?

- How can these interventions be bundled and implemented for utilization?

- Will the effectiveness of these assessments and interventions differ when performed by nurses, pharmacists or physicians?

Answers to these and other important questions have the potential to greatly impact care for patients with VTE. In the meantime, clinicians and patients searching for guidance when selecting OACs in both AF and VTE treatment must look beyond the SAMe-TT2R2 score.

Funding

Grant funding from the National Heart, Lung, and Blood Institute and Pfizer/Bristol-Myers-Squib. Consulting fees from Portola, Pfizer/Bristol-Myers-Squib and Janssen.

\section{Conflict of Interest}

Dr. Barnes reports grants and personal fees from Pfizer/ Bristol-Myers Squibb, personal fees from Janssen and Portola, and grants from National Heart Lung and Blood Institute, outside the submitted work.

\section{References}

1 Schulman S, Ageno W, Konstantinides SV. Venous thromboembolism: past, present and future. Thromb Haemost 2017;117(07): 1219-1229

2 Schulman S, Singer D, Ageno W, Casella IB, Desch M, Goldhaber SZ. NOACs for treatment of venous thromboembolism in clinical practice. Thromb Haemost 2017;117(07):1317-1325

3 Lip G, Freedman B, De Caterina R, Potpara TS. Stroke prevention in atrial fibrillation: past, present and future. Comparing the guidelines and practical decision-making. Thromb Haemost 2017;117 (07):1230-1239 
4 Apostolakis S, Sullivan RM, Olshansky B, Lip GYH. Factors affecting quality of anticoagulation control among patients with atrial fibrillation on warfarin: the SAMe- $\mathrm{TT}_{2} \mathrm{R}_{2}$ score. Chest $2013 ; 144$ (05):1555-1563

5 Abumuaileq RR, Abu-Assi E, Raposeiras-Roubin S, et al. Evaluation of SAMe-TT2R2 risk score for predicting the quality of anticoagulation control in a real-world cohort of patients with nonvalvular atrial fibrillation on vitamin-K antagonists. Europace 2015;17(05):711-717

6 Lobos-Bejarano JM, Barrios V, Polo-García J, et al; on behalf the PAULA Study Team. Evaluation of SAMe-TT2R2 score and other clinical factors influencing the quality of anticoagulation therapy in non-valvular atrial fibrillation: a nationwide study in Spain. Curr Med Res Opin 2016;32(07):1201-1207
7 Lip GY, Lane DA. Stroke prevention in atrial fibrillation: a systematic review. JAMA 2015;313(19):1950-1962

8 Barco S, Granzier S, Coppens M, et al. Determinants of the quality of warfarin control after venous thromboembolism and validation of the SAMe-TT2-R2 score: an analysis of hokusai-VTE. Thromb Haemost 2019. Doi: 10.1055/s-0039-1678546

9 Kataruka A, Kong X, Haymart B, et al. SAMe-TT2R2 predicts quality of anticoagulation in patients with acute venous thromboembolism: the MAQI2 experience. Vasc Med 2017;22(03): 197-203

10 Palareti G, Antonucci E, Lip GY, et al; START-Register Participants. The SAME-TT2R2 score predicts the quality of anticoagulation control in patients with acute VTE. A real-life inception cohort study. Thromb Haemost 2016;115(06):1101-1108 